\title{
Early cardiovascular involvement in Chronic Obstructive Pulmonary Disease
}

\author{
M. Malerba, G. Romanelli
}

ABSTRACT: Early cardiovascular involvement in Chronic Obstructive Pulmonary Disease. M. Malerba, G. Romanelli.

Cardiovascular $(\mathrm{CV})$ disease represents a considerable risk factor in terms of both morbidity and mortality in elderly patients with chronic obstructive pulmonary disease (COPD). In fact, there is a considerable evidence of this association: for only 20 years forced expiratory volume in 1 second $\left(\mathrm{FEV}_{1}\right)$ has been considered as predictive of cardiovascular mortality especially in elderly patients. At present, the emerging evidence suggests that hypoxia, systemic inflammation, oxidative stress may cause an early sub-clinical cardiovascular involvement in patients with COPD. Aging is a selective process dramatically affecting certain portions of the cardiovascular system for example: diminished beta-adrenergic responsiveness, increased myocardial and vascular stiffness, decreased arterial baroreflex, vagal outflow and compromission of diastolic function. The nature of these interactions is complex and involves not only mechanisms of aging but also multiple defined and undefined (e.g., genetic) risk factors. Cardiovascular diseases are the main causes of mortality among the subjects with impaired lung function. Even mild reductions in expiratory flow volumes amplify the risk of ischemic heart diseases, strokes, and sudden cardiac deaths 2-to 3-fold, independent of other risk factors. The mechanism or mechanisms responsible for this association, however, remain largely unknown.

Monaldi Arch Chest Dis 2009; 71: 2, 59-65.

Keywords: Chronic obstructive pulmonary disease (COPD), Early cardiovascular involvement.

Department of Internal Medicine, University of Brescia, Italy.

Correspondence: Mario Malerba, Department of Internal Medicine, University of Brescia, la Divisione di Medicina, Spedali Civili, P.zza Spedali Civili 1, 25100 Brescia, Italy; e-mail: malerba@med.unibs.it

\section{Introduction}

Chronic obstructive pulmonary disease (COPD) is characterised by a not completely reversible airflow obstruction due to a complex pathology involving large and small airways, lung parenchyma and the pulmonary vasculature [1]. COPD affects over the $5 \%$ of the adult population and it is a major cause of increasing mortality and morbidity worldwide [2]. Recently a compelling body of evidence highlighted that COPD is not just a lung disease, but a systemic disease, with multiple effects on the whole organism and the cardiovascular system in particular, involving mainly atherosclerosis and promoting diseases such as myocardial infarction and arrhythmias, stroke and systemic artheriopathies [3]. A remarkable high morbidity due to cardiovascular diseases in COPD patients has been observed at the present time and the most evident explanation for this high prevalence is the elevated occurrence among COPD patients of smoking and other known risk factors for cardiovascular diseases such as sedentary lifestyle and poor diet. However, several studies have shown that impaired lung function is associated with cardiovascular risk even after adjusting for known cardiovascular risk factors including age, sex, smoke and cholesterol [4] and that the main causes of death among COPD patients are of cardiovascular origin. Recent studies [5] indicate that reduced $\mathrm{FEV}_{1}$ is an independently important risk factor for cardiovascular mortality, showing that among the lower and the higher quintile of $\mathrm{FEV}_{1}$ the risk of death for cardiovascular cause is increased approximately by $75 \%$ in both women and men. Moreover, airflow obstruction as measured by a reduced $\mathrm{FEV}_{1} / \mathrm{FVC}$ ratio may be an important modifier effect: when the combination of reduced $\mathrm{FEV}_{1} / \mathrm{FVC}$ and arrhythmias is observed the risk of coronary events is increased by over twofold. This increased morbidity and mortality may represent just the "tip of the iceberg" of unknown early cardiovascular alterations in COPD patients.

This evidence encourages early cardiovascular risk stratification of COPD patients in the daily clinical practice. Nevertheless, specialists often focus on their own field when considering the patient, and the presence of systemic involvement in COPD patients is not taken into account or poorly reported. Moreover, the possible risks of some treatments are underscored and the prevention of cardiovascular diseases is inadequate in these patients.

\section{Pathogenetic factors}

\section{Systemic inflammation}

Recent research has demonstrated the existence of a relationship between chronic obstructive 
pulmonary disease (COPD) and systemic inflammation [6]. In fact, cigarette smoke is able to induce an activation of the systemic inflammatory cascade in susceptible subjects; following the cessation of smoking, a number of subjects have shown a decrease in inflammation, however in many cases inflammation becomes self-perpetuating [7].

A reduced $\mathrm{FEV}_{1}$, coupled with an active smoking status, has an influence on the levels of C-Reactive Protein (CRP), a common marker of systemic inflammation [8]. CRP, which is now considered a major risk indicator for cardiovascular disease [9], has been found to be higher even in patients with stable COPD, and may be an important factor in the progression of atherosclerosis in these patients [5].

Atherosclerosis, in fact, is considered to have an important inflammatory component [10, 11]. CRP, in particular, upregulates the production of pro-inflammatory cytokines and tissue factors in monocytes, it increases LDL uptake in macrophages and directly induces the expression of adhesion molecules in endothelial cells [12]. In addition to this, CPR may also deposit directly in the arterial wall during the atherogenetic process, interacting with other inflammatory mediators to create foam cells, which serve as the building blocks of the atherogenetic plaques [13]. On the other hand, serum fibrinogen may also promote atherosclerosis, by increasing blood viscosity and acting as a co-factor for platelets aggregation [14]. There is also data demonstrating the possible role of acute lung in- flammation in the induction of acute cardiac events, by acting on the coagulation cascade through the elevation of plasma fibrinogen and IL-6 levels, as found in COPD exacerbations [15] (figure 1).

Statins have been shown to benefit both cardiovascular disorders (CVD) and COPD, with a probable effect on the inflammation in the lungs and in the atheromatous plaques [16]. On the contrary inhaled corticosteroids have not had significant effect on cardiovascular mortality in COPD [17].

\section{Oxidative stress}

COPD is an inflammatory lung disease characterised by both systemic and local chronic inflammation and oxidative stress [18]. Among the various sources of oxidative stress, the increased burden of inhaled oxidants stands out as a prominent cause of lung damage, because COPD is frequently caused by long-term inhalation of noxious gases and particles, such as cigarette smoke[19]. As a matter of fact, long-term smoke exposure can result in a dramatic systemic oxidant-antioxidant imbalance as reflected by an increase in lipid peroxidation products and depleted levels of antioxidants like vitamins A and C in smoking patient's plasma [20].

The increase in reactive oxygen species (ROS), generated by various inflammatory, immune and epithelial cells of the airways, is reflected by a parallel increase in markers of oxidative stress in the airspaces, sputum, breath, lungs and blood in patients with COPD.

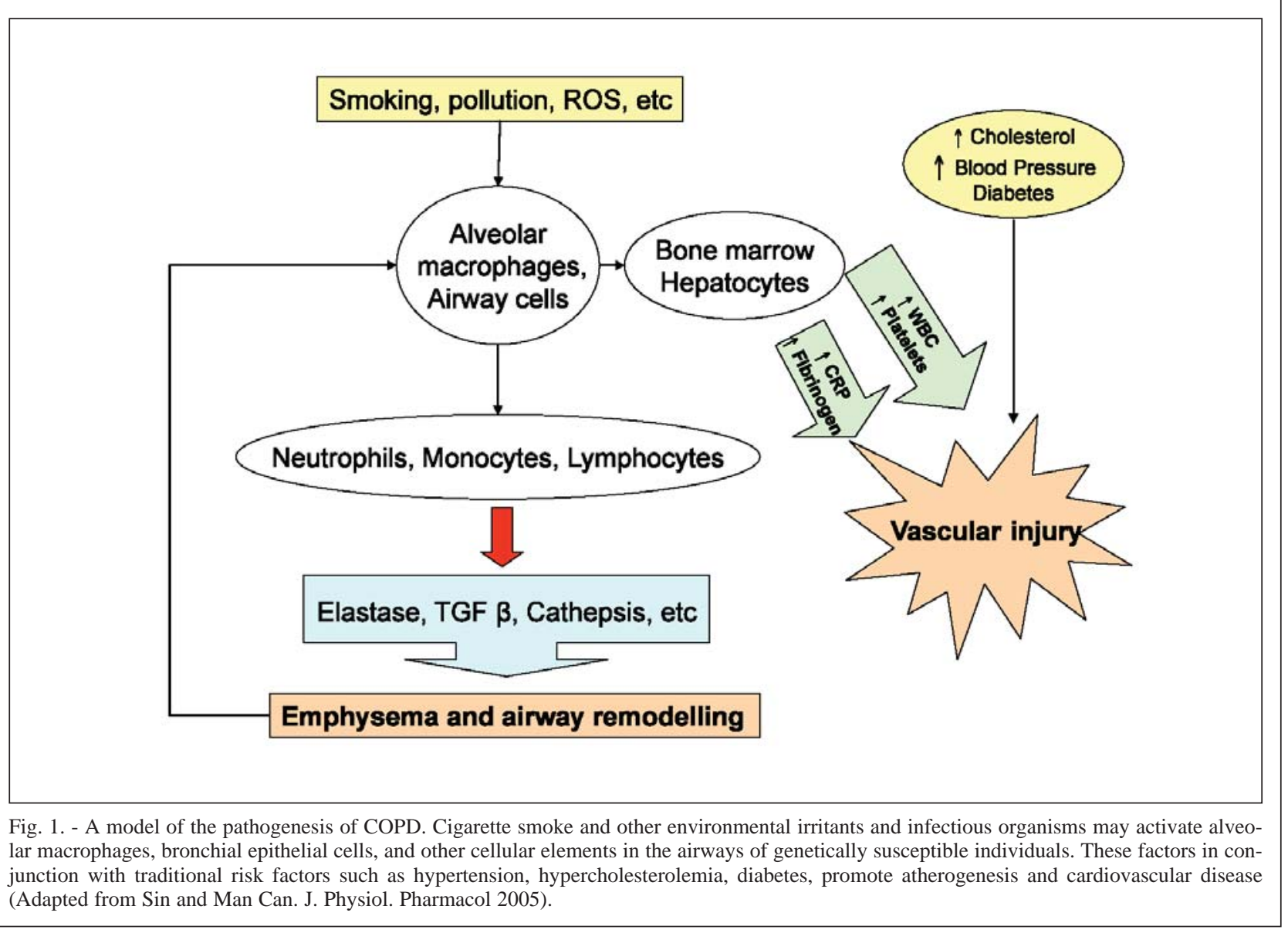


The presence of elevated amounts of ROS may play a role in enhancing the inflammatory response in COPD through the activation of stress kinases and redox sensitive transcription factors. Recent data has indicated that oxidative stress and pro-inflammatory mediators can alter nuclear histone acetylation, allowing access for transcription factors to specific DNA binding sites, leading to enhanced pro-inflammatory gene expression in various lung cells. Thus, the presence of oxidative stress may have important consequences on several events of lung physiology and for the pathogenesis of COPD [20]. Such consequences include increased sequestration of neutrophils in the pulmonary microvascular vessels, oxidative inactivation of antiproteases and surfactants, hypersecretion of mucus, membrane lipid peroxidation, alterations of mitochondrial respiration, alveolar epithelial injury, remodelling of extracellular matrix and finally apoptosis [21].

The impact of ROS on chromatin regulation may have a role in the poor efficacy of corticosteroids in COPD. Consequently, an effective, wide spectrum antioxidant therapy, with the characteristics of adequately high bioavailability and potency, is urgently needed to control the localised oxidative and inflammatory processes that occur in the pathogenesis of COPD [22].

\section{Hypoxia}

The biological response to chronic hypoxia is a systemic, very complex, as-of-yet unclear mechanism, based on the control of genetic expression.
The heart subsists on an obligate aerobic metabolism and, at rest, consumes 10-15 ml of oxygen per minute for $100 \mathrm{~g}$ of tissue, a rate higher than that of the brain. Therefore, a constant and adequate oxygen supply is necessary for the heart to stay functioning and vital [23].

Oxygen is also the main determinant of genetic expression in myocardial cells: in a situation of deficitary oxygen supply, the genetic expression patterns of these cells becomes significantly alterated [24]. Furthermore, oxygen is fundamental in the formation of nitric oxide (NO), which contributes, along with many other factors, to control vascular tone and, consequently, the blood supply to the tissues [25]. In addition, oxygen of course has a central role in the production of ROS, which may induce irreversible damage to cells [25]. Chronic hypoxia induces a number of clinically relevant modifications, both structural and functional, in the organism, of which polyglobulia and the remodelling of pulmonary vessels are well known examples [26]. With this in mind, the elevated frequency of left ventricular hypertrophy and coronaropathy, documented in hypoxemic COPD patients, could undoubtedly be imputed to chronic hypoxia [27].

While it is unclear whether chronic hypoxia is a direct cause of cardiac dysfunction, as opposed to only being the trigger of an inadequate adaptive response, it is still surely recommended, to attempt a correction of this consequence of COPD in everyday clinical practice, in order to reduce its negative impact, both on a cardiovascular and systemic level (figure 2).

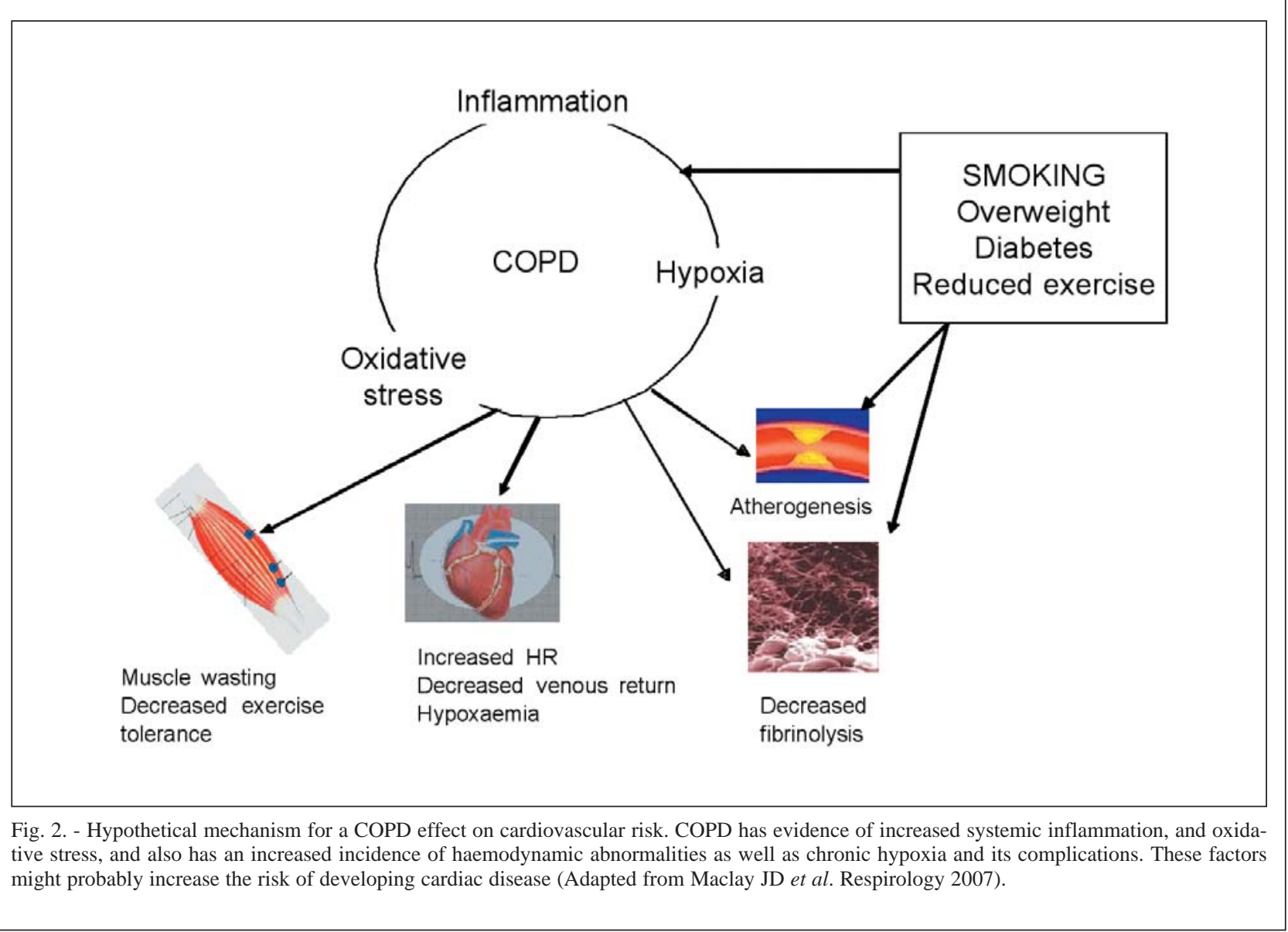




\section{Aging and atherosclerosis}

Aging is an important determinant of both arterial stiffening and the development of emphysema, and it is also a key cause of skin wrinkling and osteoporosis [33, 34]. Consequently, there has been some interest in aging as a mechanism of disease in patients with COPD [35]. Although cigarette smoke exposure is the main cause of COPD, individuals vary in their susceptibility to its effects, in fact many of the features of COPD are also seen with normal aging among never-smokers. Progressive decline in lung function, characteristic feature of COPD, for example, also occurs with age in healthy individuals [36]. Aging lungs also show progressive distal airspace enlargement, associated with elastin fibrin fragmentation and loss of elasticity, resulting in an emphysema-like condition [33, 37].

Aging is characterised by shortening of the DNA component of telomeres, the specialised segments located at the end of eukaryotic chromosomes which protect them from degradation and recombination [38]. In most somatic cells telomeres shorten with every cell cycle, and systemic oxidative stress and inflammation enhance this shortening process [39]. Telomere length therefore reflects replication history of cells, but it is also a reflection of cumulative oxidative stress and chronic inflammation acting on progenitor cells [40] and provides a marker of biological age (figure 3 ).

Cellular senescence is associated with shortened or damaged telomeres and it is characterised by permanent exit from the cell cycle, morphologic changes, and altered function. Senescent cells show increased release of cytokines and chemokines and enhanced matrix metalloprotease activity [41], which are potential mechanisms for the enhanced inflammation and tissue destruction in emphysema. Accelerated aging, as measured by telomere shortening has also been linked to cardiovascular disease [42]. Telomere length is a predictor of cardiovascular events [43], and reduced leukocyte telomere length is associated with all cause mortality in patients with stable coronary disease [42]. Moreover shorter telomeres have been detected in senescent endothelial cells and vascular smooth muscle cells from human atherosclerotic plaques [44-47]. Senescent cells promote endothelial dysfunction and hence atherosclerosis, and appear to be implicated in plaque destabilisation $[46,47]$. Therefore, accelerated aging, characterised by shortening of the DNA component of telomeres, might cause vascular and pulmonary disease by an increased systemic inflammation, connective tissue destruction, and endothelial dysfunction. Accelerated aging processes may therefore be the link for the association between COPD and increased cardiovascular risk.

The effect of age on heart size has been assessed by several techniques, including echocardiography. Most cross-sectional studies have demonstrated that end-diastolic and end-systolic left-ventricle (LV) wall thickness and estimated of left-ventricular mass, measured via M-mode echocardiography, increase with age.

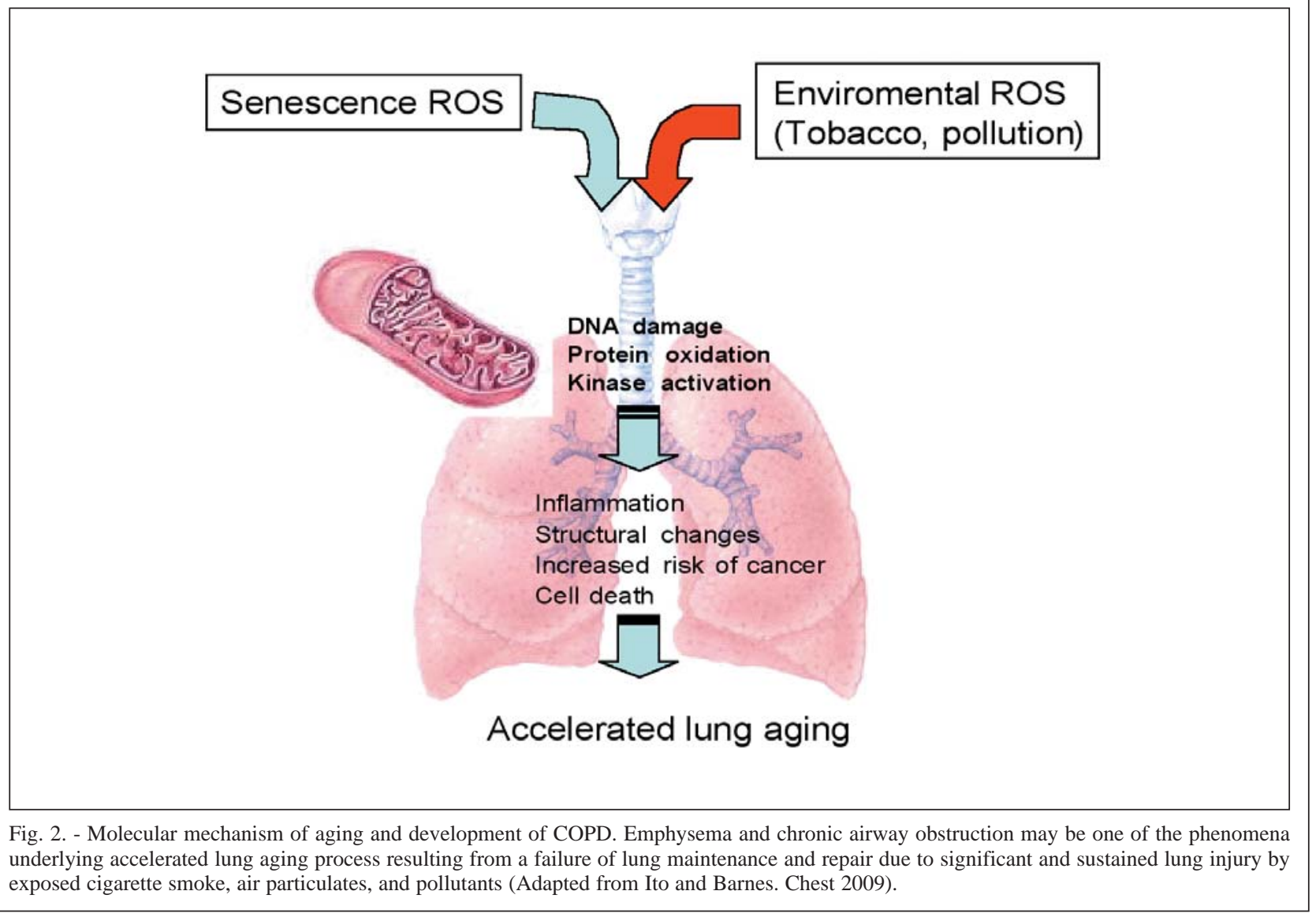




\section{Cardio-vascular dysfunction}

According to multiple studies, involving different patient series, an approximation of 10 to $40 \%$ of subjects with COPD also show signs of left ventricular dysfunction, and a significantly high percentage of deaths in COPD patients is actually attributable to a cardiovascular cause. Together with lung cancer, cardiovascular disease is the main cause of death in mild to moderate COPD patients, while respiratory failure is responsible of the death of most moderate to severe $\left(\mathrm{FEV}_{1}<60 \%\right)$ COPD patients [28].

However, a meta-analysis conducted on over 80,000 patients revealed that subjects in the lowest lung function quintile have almost a doubled risk of cardiovascular mortality, compared to subjects in the highest lung function quintile [29]. In particular, for every $10 \%$ reduction in $\mathrm{FEV}_{1}$, these patients suffer a $28 \%$ increase in cardiovascular mortality. In addition to being a prominent cause of death, cardiovascular disease is also responsible of a severe impairment in the quality of life of COPD patients, by increasing the number of hospitalisations these patients require: according to the Lung Health Study, $42 \%$ of the first, and $44 \%$ of the second hospitalisations were due to CVD in patients with mild to moderate COPD, while respiratory causes accounted for a mere $14 \%$ of admissions [30].

The knowledge of this significant data, coupled with several other studies, contributes to defining and ultimately establishing COPD as a powerful independent risk factor for cardiovascular morbidity and mortality [5].

Even after adjustments for the most common cardiovascular risk factors, COPD patients have a 4.5 risk ratio of developing chronic heart failure, compared to age-matched non-COPD controls [31]. However, the association of CVD and COPD is often overlooked: a recent study has evidenced how, of the 405 COPD patients involved, a remarkable $20.5 \%$ (83 patients) were diagnosed with concomitant heart failure following a systemic diagnostic investigation [32].

The interaction between lungs and heart due to their anatomical and functional relation produces mainly two types of consequences: diseases sharing similar risk factors, such as cigarette smoke and atherosclerosis (e.g. coronary, carotid and peripheral artery disease) or heart failure and COPD; secondly illness resulting in dysfunction of heart function due to lung diseases such as pulmonary hypertension and ventricular dysfunction secondary to increased intra-thoracic mechanical load.

In fact, COPD patients have an increased risk of fatal myocardial infarction even independent of smoking status, this encourages the search of atherosclerotic heart disease in these patients with exercise tests or other more complex and expensive methods [48].

A link between COPD and cardiovascular disease comes from the measures of central arterial stiffness a new technique for assessing cardiovascular risk [49]. This measure (termed pulse wave velocity) reflects more closely the pathological state of the central arteries and therefore appears to be better associated with coronary artery disease and with smoking injury [50]. A relationship between pulse wave velocity and $\mathrm{FEV}_{1} \%$ predicted has been described in a study with COPD patients, in which patients with a $\mathrm{FEV}_{1} \%$ predicted $<50$ had increased arterial stiffness, compared to patients with $\mathrm{FEV}_{1} \%$ predicted > 50 [49].

It is now believed that the presence of heart failure is overlooked in patients with COPD [51], however the exact prevalence of heart dysfunction in these patients is not yet known [51]. One study has demonstrated a prevalence of left ventricular failure of $20 \%$ among COPD patients who have not previously had this diagnosis [52]. Diagnosing heart failure in the presence of COPD is complicated by similarities in symptoms and physical findings $[53,54]$. The chest X-ray is less sensitive for detecting heart failure in these patients because of hyper-inflated lungs, and heart dilatation caused by COPD itself. Moreover, in severe COPD some degree of pulmonary congestion is usually present on chest X-ray [55]. Electrocardiographic abnormalities may overlap in COPD and heart failure patients [56]. Plasma B-type natriuretic peptide (BNP) can help the physician in differentiating COPD with and without heart failure [57]. However during COPD exacerbations, BNP can be elevated, although not as high as in severe heart failure, due to pressure or volume overload acting on right ventricle [58], thus producing confounding results in a large number of patients. The assessment of left ventricular function by two dimensional echo-Doppler examination is usually required to demonstrate or exclude heart failure, however in COPD patients echocardiographic windows are often limited by hyper-inflated lungs and precise measurements can be complex in up to 10-30\% of COPD patients [59]. To avoid these difficulties cardiovascular magnetic resonance or radionuclide ventriculography may be required, however disadvantages of these procedures are the time-consuming data acquisition and post processing and the higher cost compared to echocardiography [60].

In our personal clinical experience, we studied a cohort of twelve COPD patients with stable mild airflow limitation and without cardiovascular comorbidity. At echocardiography assessment ten patients $(83 \%)$ showed a first degree diastolic dysfunction of the left ventricle (unpublished data). As a possible hypothesis, a not completely reversible respiratory obstruction may be associated to a sub-clinical diastolic dysfunction of the left ventricle, which may be an early sign of occult coronaropathy. Currently, the role of systemic inflammation in promoting the atherosclerotic process is well known, thus it may also be possible that unrecognised vascular alterations could lead to asymptomatic heart modifications in these patients, which could be suspected whenever an altered left ventricle function is found in echocardiography. Finally, considering the cardio-pulmonary system altogether, it is certainly possible that alterations in the respiratory mechanics may 
induce modifications of the cardiac function, left ventricle included. Further studies will be necessary to assess the intricate pathophysiological interactions between heart, lungs, and the organism in its entirety.

In conclusion, there is considerable interest in comorbidities associated with COPD, and especially the significant burden of morbidity and mortality associated with CVD. Patients with COPD are subjected to stressors including cigarette smoke, chronic inflammation, oxidative stress and hypoxia as well as repeated exacerbations with marked acute local and systemic inflammation and hypoxia, one might expect cardiovascular complications. Because these extra-pulmonary manifestations of COPD are common and may have relevant implications for the patient prognosis, a precise screening and appropriate treatment of these conditions is compulsory. Observational studies support this supposition and currently, there are a number of ongoing studies investigating the pathogenesis of atherosclerosis correlate to airways disease. In the next future it will be essential to assess whether COPD is able to influence atherosclerosis and cardiac events separately from smoking and above all whether anti-COPD interventions, other than smoking cessation, can modify cardiovascular risk in these patients.

Given the complex nature of both COPD and CVD, it seems likely that no single cause of the association will be found but in the near future we can expect a better understanding of the mechanisms connecting these two conditions as well as a clearer view on the most relevant targets for therapeutic intervention.

\section{References}

1. Pauwels RA, Buist AS, Calverley PM, et al. Global Strategy for the Diagnosis, Management, and Prevention of Chronic Obstructive Pulmonary Disease. NHLBI/WHO Global Initiative for Chronic Obstructive Lung Disease (GOLD) workshop summary. Am J Respir Crit Care Med 2001; 163: 1256-76.

2. Chen JC, Mannino DM. Worldwide epidemiology of chronic obstructive pulmonary disease. Curr Opin Pulm Med 1999; 5: 93-9.

3. Sin DD, Man SFP. Chronic obstructive pulmonary disease: a novel risk factor for cardiovascular disease. Can J Physiol Pharmacol 2005; 83: 8-13.

4. Maclay D, McAllister DA, MacNee W. Cardiovascular risk in chronic obstructive pulmonary disease. Respirology 2007; 12: 634-641.

5. Sin DD, Man SFP. Chronic Obstructive Pulmonary Disease as a risk factor for cardiovascular morbidity and mortality. Proc Am Thorac Soc 2005; 2: 8-11.

6. Gan WQ, Man SF, Sin DD, et al. Association between chronic obstuctive pulmonary disease and systemic inflammation: a systematic review and a meta-analysis. Thorax 2004; 59: 574-580.

7. Yanbaeva DG, Dentener MA, Creutzberg EC, et al. Systemic effects of smoking. Chest 2007; 131: 1557-1566.

8. Gan WQ, Man SF, Sin DD. The interactions between cigarette smoking and reduced lung function on systemic inflammation. Chest 2005; 27: 558-564.

9. Ridker PM. Clinical application of C-reactive protein for cardiovascular disease detection and prevention. Circulation 2003; 107: 363-369.
10. Ross R. Atherosclerosis - an inflammatory disease. $N$ Engl J Med 1999; 340: 115-126.

11. Lusis AJ. Atherosclerosis. Nature 2000; 407: 233-241.

12. Pasceri V, Willerson JT, Yet ETH. Direct proinflammatory effect of C-reactive protein on human endothelial cells. Circulation 2000; 102: 2165-2168.

13. Zwaka TP, Hombach V, Torzewski J. C-reactive protein-mediated low density lipoprotein uptake by macrophages: implications for atherosclerosis. Circulation 2001; 103: 1194-1197.

14. Danesh J, Collins R, Appleby P, et al. Association of fibrinogen, C-reactive protein, albumin, or leukocyte count with coronary heart disease: meta-analyses of prospective studies. JAMA 1998; 279: 1477-1482.

15. Wedzicha JT, Seemungal TA, MacCallum PK, et al. Acute exacerbations of chronic obstructive pulmonary disease are accompanied by elevations of plasma fibrinogen and serum IL6 levels. Thromb Haemost 2000; 84: 210-215.

16. Mancini GBJ, Etminan M, Zhang B, Levesque LE, et al. Reduction of morbidity and mortality by statins, angiotensin-converting enzyme inhibitors, and angiotensin receptor blockers in patients with Chronic Obstructive Pulmonary Disease. J Am Coll Cardiol 2006; 47: 2554-2560.

17. Calverly PMA, Anderson JA, Celli B, et al. Salmeterol and Fluticasone Propionate and Survival in Chronic Obstructive Pulmonary Disease. N Engl J Med 2007; 356: 775-789.

18. Rahman I. Oxidative stress in pathogenesis of chronic obstructive pulmonary disease: cellular and molecular mechanisms. Cell Biochem Biophys 2005; 43: 167-88.

19. Rahman I. Oxidative stress and gene trascription in asthma and chronic obstructive pulmonary disease: antioxidant terapeutic targets. Curr Drug Inflamm Allergy 2002; 1: 219-315.

20. Rahman I. The role of oxidative stress in the pathogenesis of COPD: implication for therapy. Treat Respir Med 2005 4: 175-200.

21. Kirkham P, Rahman I. Oxidative stress in asthma and COPD: antioxidants as a therapeutic strategy. Pharmacol Ther 2006; 111: 476-94

22. Ito K, Barnes PJ. COPD as a disease of accelerating lung aging. Chest 2009; 135: 173-80.

23. Giordano FJ. Oxygen, oxidative stress, hypoxia and heart failure. J Clin Invest 2005; 115: 500-508.

24. Huang Y, et al. Cardiac myocyte-specific HIF-1alpha deletion alters vascularization, energy availability, calcium flux, and contractility in the normoxic heart. FASEB J 2004; 18: 1138-1140.

25. Davies KJ. Oxidative stress: the paradox of aerobic life. Biochem Soc Symp 1995; 61: 1-31.

26. Carmeliet P, et al. Role of HIF-1alpha in hypoxia-mediated apoptosis, cell proliferation and tumour angiogenesis. Nature 1998; 394: 485-490.

27. Khatri JJ, Johnson C, Magid R, et al. Vascular oxidant stress enhances progression and angiogenesis of experimental atheroma. Circulation 2004; 109: 520-525.

28. Sin DD, Anthonisen NR, Soriano JB, Agusti AG. Mortality in COPD: role of comorbidities. Eur Respir $J$ 2006; 28: 1245-1257.

29. Sin DD, Wu L, Man SF. The relationship between reduced lung function and cardiovascular mortality: a population-based study and a systematic review of the literature. Chest 2005; 127: 1952-1959.

30. Anthonisen NR, Connet JE, Enright PL, et al. Hospitalization and mortality in the Lung Health Study. Am J Respir Crit Care Med 2002: 333-339.

31. Le Jemtel TH, Padeletti M, Jelic S. Diagnostic and therapeutic challenges in patients with coexistent chronic obstructive pulmonary disease and chronic heart failure. J Am Coll Cardiol 2007; 49: 171-180. 
32. Rutten FH, Moons KG, Cramer MJ, et al. Recognising heart failure in elderly patients with stable chronic obstructive pulmonary disease in primary care: cross sectional diagnostic study. BMJ 2005; 331: 1379-1382.

33. Janssens JP, Pache JC, Nicod LP. Physiological changes in respiratory function associated with ageing. Eur Respir J 1999; 13: 197-205.

34. O'Rourke M. Arterial stiffness, systolic blood pressure, and logical treatment of arterial hypertension. Hypertension 1990; 15: 339-347.

35. Vogelmeier C, Bals R. Chronic obstructive pulmonary disease and premature aging. Am J Respir Crit Care Med 2007; 175: 1217-1218.

36. Fletcher C, Peto R. The natural history of chronic airflow obstruction. BMJ 1977; 1: 1645-1648.

37. Teramoto S, Ishii M. Aging, the aging lung, and senile emphysema are different. Am J Respir Crit Care Med 2007; 175: 197-198.

38. Chan SR, Blackburn EH. Telomeres and telomerase. Philos Trans R Soc Lond B Biol Sci 2004; 359: 109121.

39. Saretzki G, Von Zglinicki T. Replicative aging, telomeres, and oxidative stress. Ann NY Acad Sci 2002; 959: 24-29.

40. Aviv A. Telomeres and human somatic fitness. $J$ Gerontol A Biol Sci Med Sci 2006; 61: 871-873.

41. Nyunoya T, Monick MM, Klingelhutz A, Yarovinsky TO, Cagley JR, Hunninghake GW. Cigarette smoke induces cellular senescence. Am J Respir Cell Mol Biol 2006; 35: 681-688.

42. van der Harst P, van Veldhuisen DJ, Samani NJ. Expanding the concept of telomere dysfunction in cardiovascular disease. Arterioscler Thromb Vasc Biol 2008; 28: 807-808.

43. Brouilette SW, Moore JS, et al. Telomere length, risk of coronary heart disease, and statin treatment in the West of Scotland Primary Prevention Study: a nested casecontrol study. Lancet 2007; 369: 107-114.

44. Aviv H, Khan MY, Skurnick J, et al. Age dependent aneuploidy and telomere length of the human vascular endothelium. Atherosclerosis 2001; 159: 281-287.

45. Ogami M, Ikura Y, Ohsawa M, et al. Telomere shortening in human coronary artery diseases. Arterioscler Thromb Vasc Biol 2004; 24: 546-550.

46. Minamino T, Kourembanas S. Mechanisms of telomerase induction during vascular smooth muscle cell proliferation. Circ Res 2001; 2001: 237-243.
47. Tsirpanlis G. Cellular senescence, cardiovascular risk, and CKD: a review of established and hypothetical interconnections. Am J Kidney Dis 2008; 2008: 131-144.

48. Agusti A, Soriano JB. COPD as a systemic disease. COPD 2008; 5: 133-138.

49. Sabit R, Bolton CE, Edwards PH, et al. Arterial stiffness and osteoporosis in chronic obstructive pulmonary disease. Am J Respir Crit Care Med 2007; 175: 1259-65.

50. Tillin T, Chambers J, Malik I, Coady E, et al. Measurement of pulse wave velocity: site matters. $J$ Hypertens 2007; 25: 383-9.

51. Rutten FH, Cramer MJ, Lammers JW, Grobbee DE, Hoes AW. Heart failure and chronic obstructive pulmonary disease: An ignored combination? Eur J Heart Fail 2006; 8: 706-711.

52. McCullough PA, Hollander JE, Nowak RM, et al. Uncovering heart failure in patients with a history of pulmonary disease: rationale for the early use of B-type natriuretic peptide in the emergency department. Acad Emerg Med 2003; 10: 198-204.

53. Remes J, Miettinen H, Reunanen A, Pyörälä K. Validity of clinical diagnosis of heart failure in primary health care. Eur Heart J 1991; 12: 315-21.

54. Rao BS, Cohn KE, Eldridge FL, Hancock EW. Left ventricular failure secondary to chronic pulmonary disease. Am J Med 1968; 45: 229-41.

55. Gehlbach BK, Geppert E. The pulmonary manifestations of left heart failure. Chest 2004; 125: 669-82.

56. Harrigan RA, Jones K. ABC of clinical electrocardiography. Conditions affecting the right side of the heart. BMJ 2002; 324: 1201-4.

57. Morrison LK, Harrison A, Krishnaswamy P, Kazanegra R, Clopton P, Maisel A. Utility of a rapid B-natriuretic peptide assay in differentiating congestive heart failure from lung disease in patients presenting with dyspnea. $J$ Am Coll Cardiol 2002; 39: 202-9.

58. Lang CC, Coutie WJ, Struthers AD, Dhillon DP, Winter JH, Lipworth BJ. Elevated levels of brain natriuretic peptide in acute hypoxaemic chronic obstructive pulmonary disease. Clin Sci (Lond) 1992; 83: 529-3.

59. Boussuges A, Pinet C, Molenat F, et al. Left atrial and ventricular filling in chronic obstructive pulmonary disease. An echocardiographic and Doppler study. Am J Respir Crit Care Med 2000; 162: 670-5.

60. Pennell DJ, Sechtem UP, Higgins CB, et al. Clinical indications for cardiovascular magnetic resonance (CMR): consensus panel report. Eur Heart J 2004; 25: 1940-65.

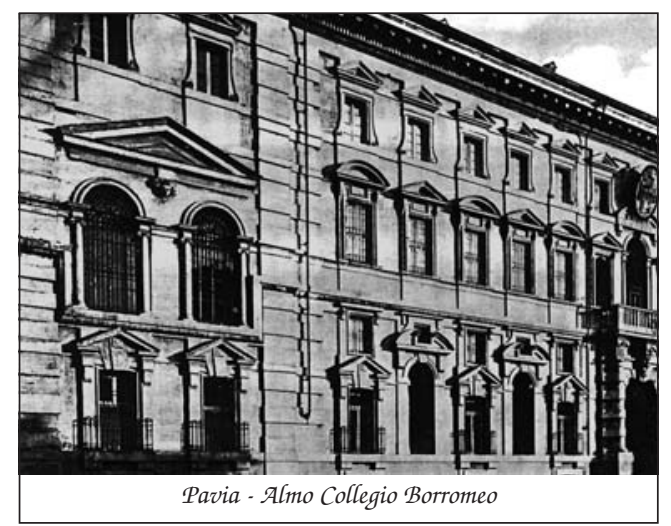

